\title{
1 Is it reasonable to account for population structure in genome-wide association studies?
}

4 Noble Research Institute LLC, Ardmore, Oklahoma, USA

$5 \quad$ Email address: bkim@noble.org

7 Abstract

8 Population structure is widely perceived as a noise factor that undermines the quality of association 9 between an SNP variable and a phenotypic variable in genome-wide association studies (GWAS).

10 The linear model for GWAS generally accounts for population-structure variables to obtain the 11 adjusted phenotype which has less noise. Its result is known to amplify the contrast between 12 significant SNPs and insignificant SNPs in a resultant Manhattan plot. In fact, however, conventional GWAS practice often implements the linear model in an unusual way in that the population-structure variables are incorporated into the linear model in the form of continuous variables rather than factor variables. If the coefficients for population-structure variables change across all SNPs, then each SNP variable will be regressed against a differently adjusted phenotypic variable, making the GWAS process unreliable. Focusing on this concern, this study investigated whether accounting for population-structure variables in the linear model for GWAS can assure the adjusted phenotypes to be consistent across all SNPs. The result showed that the adjusted phenotypes resulting across all SNPs were not consistent, which is alarming considering conventional GWAS practice that accounts for population structure. 


\section{Introduction}

Genome-wide association studies (GWAS) aim to identify single nucleotide

27 polymorphisms (SNPs) whose allelic variation is significantly tied to phenotypic variation. In principle, the tie between the allelic variation and phenotypic variation can be measured based on the variance among the phenotypic averages for all scores per each SNP (Kim, 2017; Kim, 2018a). Greater variance indicates a stronger tie. Conventional GWAS practice has been largely conducted using statistical methods such as the linear model and the linear mixed model (LMM). To date, the use of the LMM has been widely encouraged because of the general perception that accounting for a kinship matrix can reduce the noise between a phenotypic variable and an SNP variable, by correcting the bias that genetic relationship among entities in a population introduces ( $\mathrm{Yu}$ et al, 2006; Bradbury et al, 2007; Kang et al, 2008; Lipka et al, 2012; Hoffman, 2013; Kim et al, 2018b). Recently, however, Kim (2019) demonstrated that the use of a kinship matrix actually makes the LMM unreliable. In this regard, this study excluded the LMM.

Conventional GWAS practice based on the linear model often regresses each SNP variable along with population-structure variables against a phenotypic variable, one by one across all SNPs. Therein, the use of population-structure variables aims to obtain an adjusted phenotype calculated by subtracting the estimated population-structure effect from the phenotype (Yu et al, 2006; Bradbury et al, 2007; Kang et al, 2008; Lipka et al, 2012; Hoffman, 2013; Kim et al, 2018b). For reliable GWAS practice, it is crucial to assure the adjusted phenotypes resulting across all SNPs are consistent. Otherwise, every SNP variable will be regressed against a differently adjusted phenotypic variable, which consequently confounds GWAS results. This study investigated whether accounting for population structure in the linear model for GWAS assures the adjusted

47 phenotypes resulting across all SNPs to be consistent 


\section{Materials and Methods}

\section{Rice data set}

This study used a rice data set comprising SNP data, principle component analysis (PCA) data and phenotypic data. The data set was originally used for GWAS by Zhao et al. (2011) and freely available to public at http://ricediversity.org/data/index.cfm. Therefore, more information about the data set can be found from the related paper. In the original data, 413 entities were genotyped with 36,901 SNPs. The number of SNPs was reduced to 12,983 by screening with a criterion of the minor allele frequency (MAF) of 0.1 . The phenotype chosen for this study was seed length.

\section{Statistical model}

The two linear models were established as follows:

$$
y=\mu+\beta_{1} x_{S N P}+\varepsilon
$$

$$
y=\mu+\beta_{1} x_{S N P}+\beta_{2} x_{P C A 1}+\beta_{3} x_{P C A 2}+\beta_{4} x_{P C A 3}+\beta_{5} x_{P C A 4}+\varepsilon
$$

where $y=$ the phenotypic observation; $\mu=$ the phenotypic mean; $x_{S N P}=$ the SNP variable; $x_{P C A 1}$ $=$ the PCA1 variable; $x_{P C A 2}=$ the PCA2 variable; $x_{P C A 3}=$ the PCA3 variable; $x_{P C A 4}=$ the PCA4 variable; $\varepsilon=$ the error term; $\beta_{1}=$ the coefficient for $x_{S N P} ; \beta_{2}=$ the coefficient for $x_{P C A 1} ; \beta_{3}=$ the coefficient for $x_{P C A 2} ; \beta_{4}=$ the coefficient for $x_{P C A 3} ; \beta_{5}=$ the coefficient for $x_{P C A 4}$.

Equation 1 regresses the SNP variable against the phenotypic variable. Equation 2 regresses the SNP variable along with the four PCA variables $\left(x_{P C A 1}, x_{P C A 2}, x_{P C A 3}, x_{P C A 4}\right)$ against the phenotypic variable. This means that Equation 2 regresses the SNP variable against the adjusted phenotypic variable obtained by accounting for the four PCA variables. Equation 3 highlights the adjusted phenotypic variable:

$$
y-\beta_{2} x_{P C A 1}-\beta_{3} x_{P C A 2}-\beta_{4} x_{P C A 3}-\beta_{5} x_{P C A 4}=\mu+\beta_{1} x_{S N P}+\varepsilon
$$

Equation 3 is compatible with Equation 2 and represents the adjusted phenotypic variable as $y-$ $\beta_{2} x_{P C A 1}-\beta_{3} x_{P C A 2}-\beta_{4} x_{P C A 3}-\beta_{5} x_{P C A 4}$. 


\section{Manhattan plot}

The $\mathrm{F}$ test was implemented as a significance test, from which $\mathrm{P}$ values were obtained. The $\mathrm{P}$ values transformed by $-\log _{10}$ were drawn in a Manhattan plot. It is important to note that the $\mathrm{P}$ values resulting from the linear model for GWAS are prone to genomic inflation. Prior to confirming the resultant Manhattan plot, therefore, it is necessary to calculate the genomic inflation factor $\left(\lambda_{G C}\right)$. The situation of $\lambda_{G C}>1$ indicates the genomic inflation, which means that the resultant $\mathrm{P}$ values are overly estimated compared with the $\chi^{2}$-distribution (van Iterson et al, 2017). This study adjusted the genomic inflation using the genomic control. More information about the genomic control can be found in previous studies (Devlin and Roeder, 1999; Yang et al, 2011; van Iterson et al, 2017).

\section{Integrity validation of accounting for population structure in GWAS}

Equation 3 (compatible with Equation 2) regresses each SNP variable against an adjusted phenotypic variable. As GWAS handle numerous SNPs one by one at a time, it is important to assure that the adjusted phenotypes resulting across all SNPs are consistent. Otherwise, each SNP variable will be regressed against a differently adjusted phenotypic variable. The consistency among the adjusted phenotypes resulting across all SNPs can be achieved, only if every coefficient per each PCA variable is consistent across all SNPs. To check the consistency among the adjusted phenotypes resulting across all SNPs, this study calculated Pearson coefficients between the phenotype and every adjusted phenotype.

\section{Data set and $R$ code}

All computations were conducted using R (R Core Team, 2016). The data set and R scripts used in this study are freely available at https://github.com/bongsongkim/Population.Structure.GWAS. 


\section{Results}

\section{Validation of consistency across all adjusted phenotypes}

105

Table 1 summarizes the coefficients per each PCA variable, resulting from applying all

106 SNPs to Equation 3. Figure 1 represents the estimated coefficients per each PCA variable, showing

107 large variation. Figure 2 represents the estimated Pearson correlation coefficients between the

108 phenotype and every adjusted phenotype, illustrating the adjusted phenotypes resulting across all

109 SNPs are not consistent. This means that each SNP variable is regressed against a differently

110 adjusted phenotypic variable.

113 Table 1. Summary of coefficients per each PCA variable in relation to Equation 3.

\begin{tabular}{lllllll}
\hline & Min. & $1^{\text {st }} \mathrm{Qu}$. & Median & Mean & $3^{\text {rd }} \mathrm{Qu.}$ & Max \\
\hline$\beta_{2}$ & -7.833 & -2.246 & -2.162 & -2.109 & -2.024 & 3.629 \\
$\beta_{3}$ & -4.438 & -1.097 & -1.020 & -1.007 & -0.944 & 3.271 \\
$\beta_{4}$ & -13.610 & -9.229 & -9.194 & -9.180 & -9.160 & 0.659 \\
$\beta_{5}$ & -9.308 & 3.016 & 3.087 & 3.025 & 3.121 & 8.704 \\
\hline
\end{tabular}



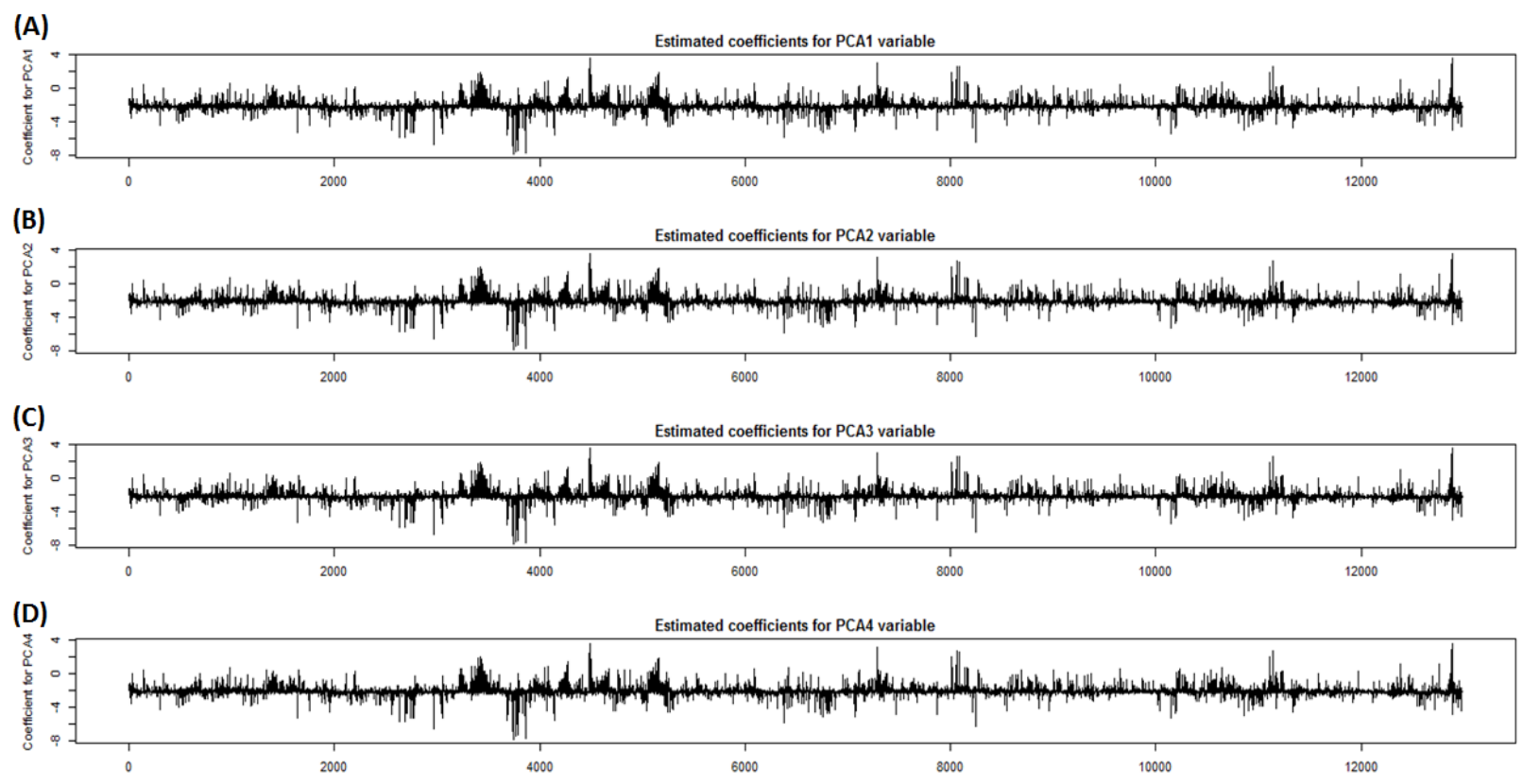

117 Figure 1. (A) Estimated coefficients for the PCA1 variable, (B) estimated coefficients for the 118 PCA2 variable, (C) estimated coefficients for the PCA3 variable, (D) estimated coefficients for 119 the PCA4 variable.

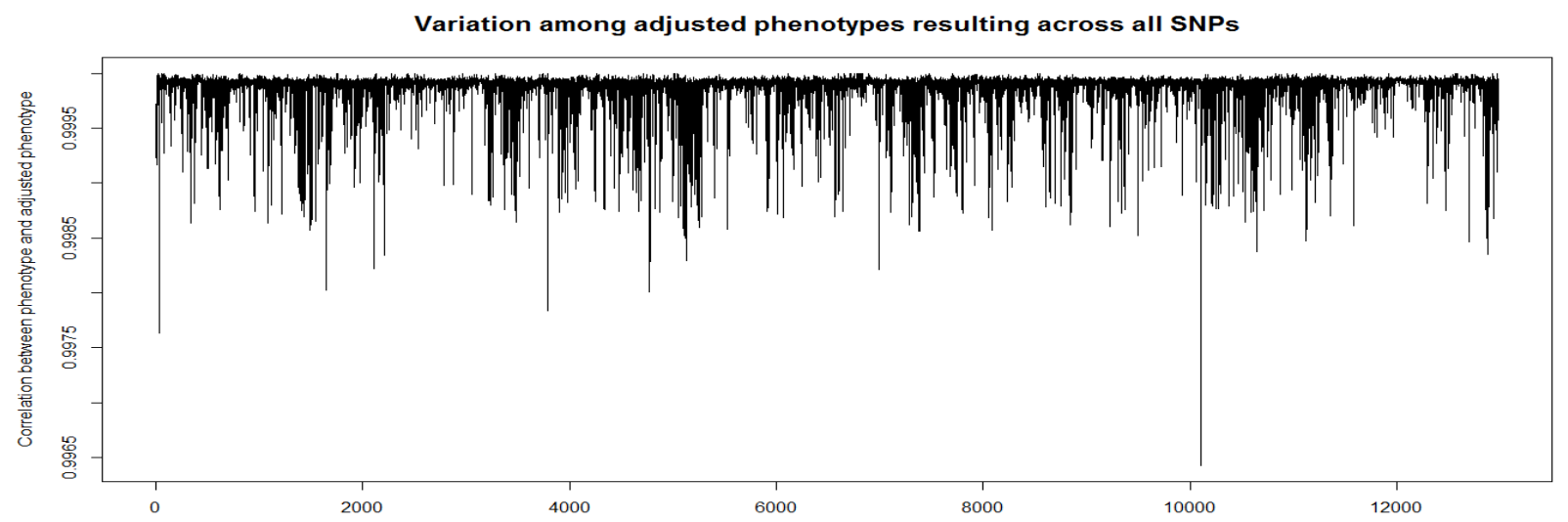

121

122 Figure 2. Pearson correlation coefficients between the phenotype and every adjusted phenotype. 


\section{Impact of accounting for population structure in GWAS}

126 Figure 3 shows four Manhattan plots, for which the same SNP and phenotypic data were used.

127 Figure 3A represents the Manhattan plot in relation to Equation 1, in which the resultant $\lambda_{G C}$ was

128 3.688. Figure $3 \mathrm{C}$ is the same as Figure $3 \mathrm{~A}$ in shape. However, Figure $3 \mathrm{C}$ meets $\lambda_{G C}=1$ by

129 implementing the genomic control with Figure 3A. Figure 3E represents the Manhattan plot in

130 relation to Equation 3, in which the resultant $\lambda_{G C}$ was 1.433. Compared with Figure 3A, Figure 3E

131 has substantially lower $\lambda_{G C}$. This suggests that accounting for the four PCA variables was

132 impactful in diminishing the genomic inflation. Figure $3 \mathrm{G}$ was obtained by adjusting Figure 3E by

133 implementing the genomic control. This led to $\lambda_{G C}=1$ in Figure 3G. It is apparent that Figure 3E

134 has clearer background than Figure 3A in relation to accounting for the four PCA variables. In this

135 regard, previous studies explained that accounting for population structure in the linear model for

136 GWAS eliminates the noise in SNP-phenotype associations, which results in clear background in

137 a resultant Manhattan plot (Yu et al, 2006; Kang et al, 2008; Korte and Farlow, 2013; Sul et al,

138 2018; Barton et al, 2019). However, Figure 4 illustrates that significant SNP-phenotype 139 associations are not consistent between Figures 3C and 3G. This means that the clear background was not from eliminating the noise in SNP-phenotype associations, but from defining new SNP141 phenotype associations. 
(A)

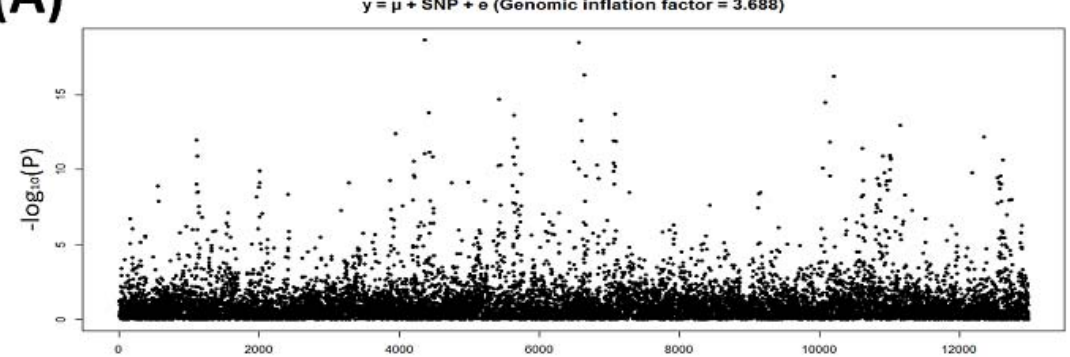

(C)

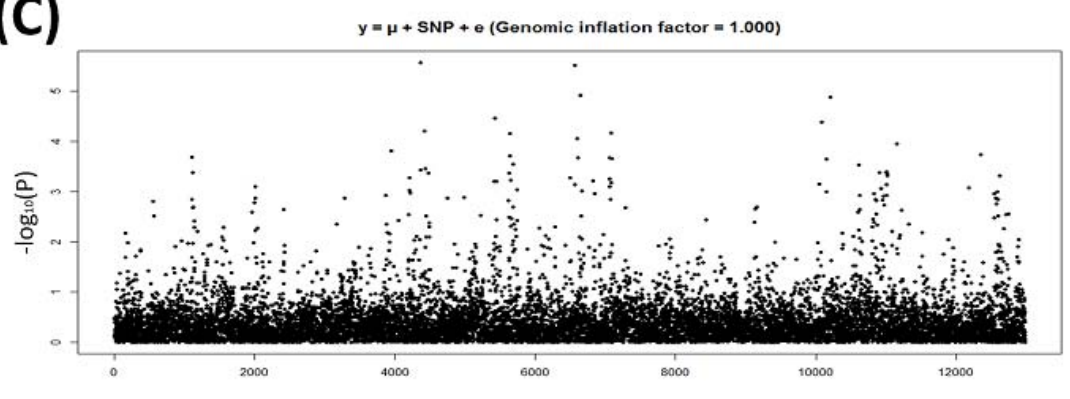

(E)

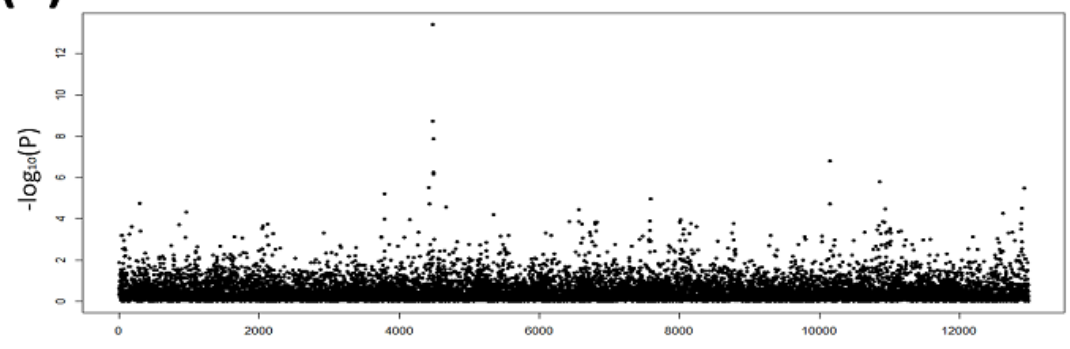

(G)

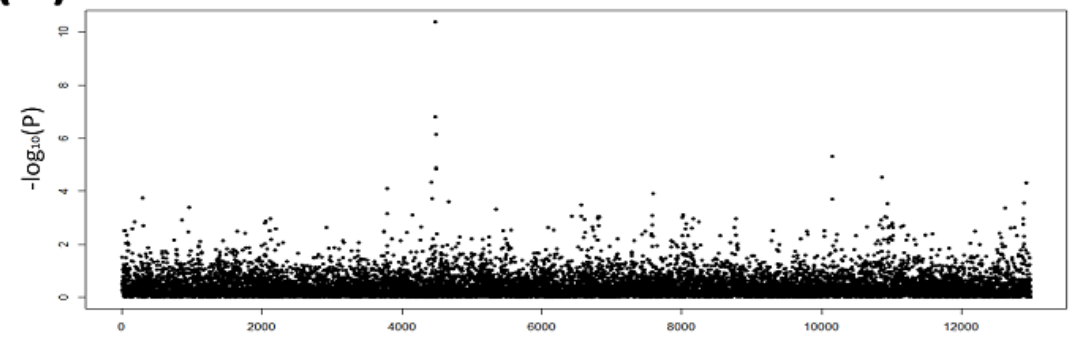

(B)

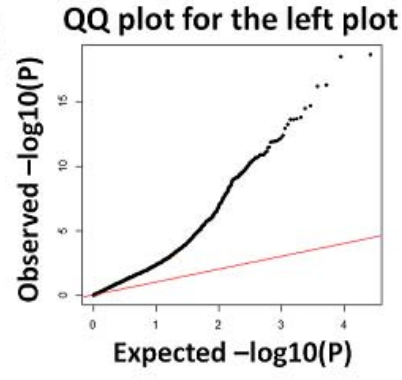

(D)

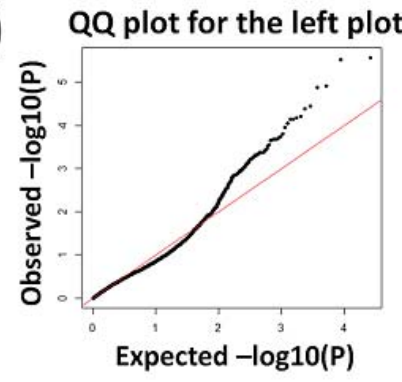

(F)

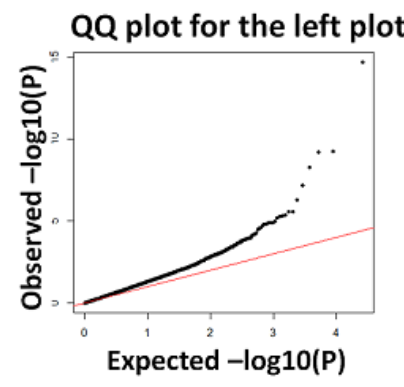

(H)

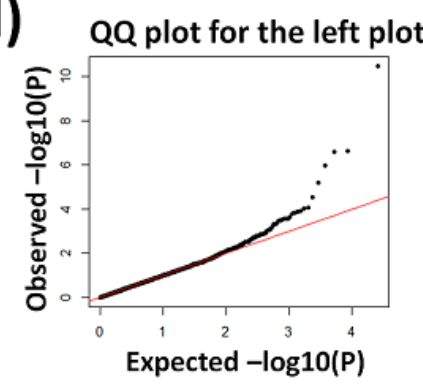

145 Figure 3. (A) Manhattan plot obtained by not accounting for the four PCA variables $\left(\lambda_{G C}=3.688\right)$,

146 (C) Manhattan plot obtained by adjusting Figure 3A with implementing the genomic control $\left(\lambda_{G C}\right.$

$147=1.000)$, (E) Manhattan plot obtained by accounting for the four PCA variables $\left(\lambda_{G C}=1.433\right),(\mathrm{G})$

148 Manhattan plot obtained by adjusting Figure $3 \mathrm{C}$ with implementing the genomic control $\left(\lambda_{G C}=\right.$ 149 1.000). 


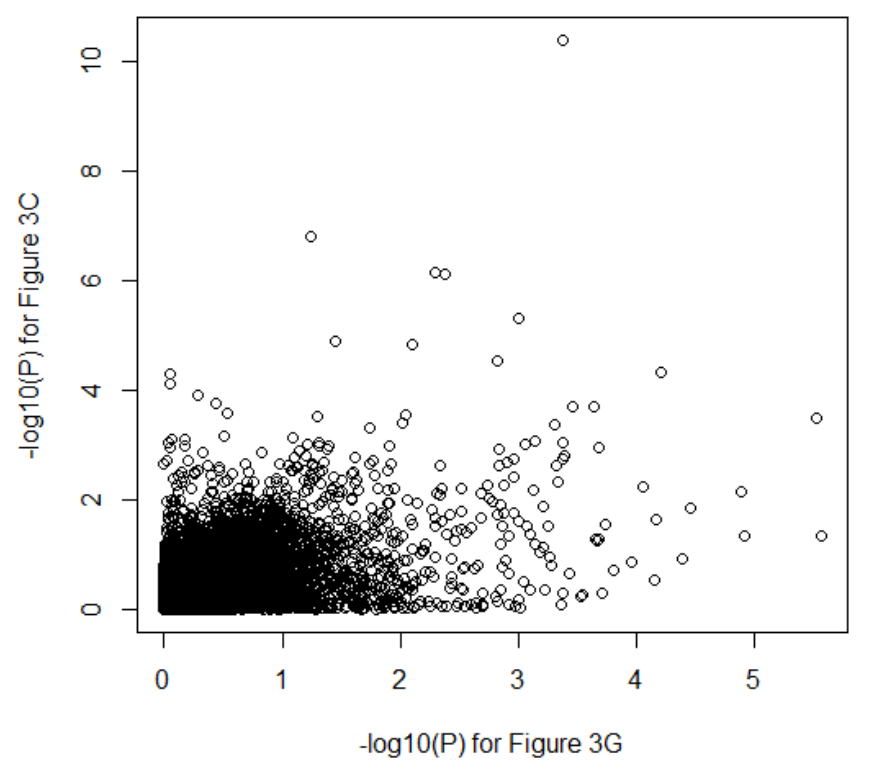

152 Figure 4. Correlation plot between the $-\log _{10}(\mathrm{P})$ values obtained by not accounting for the four 153 PCA variables (Figure $3 \mathrm{C}$ ) and the $-\log _{10}(\mathrm{P})$ values obtained by accounting for the four PCA 154 variables (Figure $3 \mathrm{G})$. 
164

165

166

167

168

169

170

171

172

173

174

175

176

177

178

179

180

181

182

183

184

185

186

187

188

189

190

191

192

\section{Discussion}

It is generally perceived that accounting for population structure in GWAS improves the quality of visual representation of a Manhattan plot by both suppressing genomic inflation and reducing false-positive SNP-phenotype associations (Yu et al, 2006; Bradbury et al, 2007; Kang et al, 2008; Lipka et al, 2012; Hoffman, 2013; Kim et al, 2018b). In fact, this study showed that accounting for the four PCA variables was very effective in diminishing the genomic inflation. Surprisingly, however, this study revealed that accounting for the four PCA variables breaks the consistency among the adjusted phenotypes resulting across all SNPs. The loss of the consistency consequently causes each SNP variable to be regressed against a differently adjusted variable, making the GWAS process unreliable. The use of population-structure variables in the linear model for GWAS implies two errors. First, the linear model is misused. Considering that the linear model is suited for analyzing data in experimental blocks, the use of continuous variables rather than factor variables necessarily causes an error. Second, the assumption for the relationship between phenotype and population structure is unjustified. The linear model for GWAS generally assumes that the population-structure variables additively contribute to the phenotypic variable. However, how the population structure biologically influences the phenotype has yet been unknown. Regardless of whether the additivity of the population-structure variables is true or false, the current way of accounting for population structure is inappropriate in that population-structure effects vary across all SNPs. The abovementioned errors consequently lead to the loss of the consistency among the adjusted phenotypes resulting across all SNPs and cause each SNP variable to be regressed against a differently adjusted phenotypic variable.

\section{Conclusion}

The linear model assures to preserve the consistency among the adjusted phenotypes resulting across all SNPs, only if factor variables such as years, locations, replications and treatments are used. This study concluded that the conventional way of accounting for population structure makes the GWAS process unreliable. This is because the population structure is represented as continuous variables. If population structure can be represented as factor variables, accounting for the population structure in the linear model for GWAS will be sound. 


\section{References}

194 Barton, Nick, Joachim Hermisson, and Magnus Nordborg. "Population Genetics: Why structure 195 matters." eLife 8 (2019): e45380.

196 Bradbury, Peter J., et al. "TASSEL: software for association mapping of complex traits in diverse 197 samples." Bioinformatics 23.19 (2007): 2633-2635.

198 Devlin, Bernie, and Kathryn Roeder. "Genomic control for association studies." Biometrics 55.4 199 (1999): 997-1004.

200 Hoffman, Gabriel E. "Correcting for population structure and kinship using the linear mixed model: 201 theory and extensions." PloS one 8.10 (2013): e75707.

202 Kang, Hyun Min, et al. "Efficient control of population structure in model organism association 203 mapping." Genetics 178.3 (2008): 1709-1723.

204 Kim, Bongsong. "Hierarchical Association Coefficient Algorithm: New Method for Genome205 Wide Association Study." Evolutionary Bioinformatics 13 (2017): 1176934317713004.

206 Kim, Bongsong. "How to Reveal Magnitude of Gene Signals: Hierarchical Hypergeometric 207 Complementary Cumulative Distribution Function." Evolutionary Bioinformatics 14 (2018a): 2081176934318797352.

209 Kim, Bongsong, et al. "GWASpro: a high-performance genome-wide association analysis 210 server." Bioinformatics (2018b).

211 Kim, Bongsong. "Is it reasonable to use a kinship matrix for best linear unbiased 212 prediction?" BioRxiv (2019): 568782.

213 Korte, Arthur, and Ashley Farlow. "The advantages and limitations of trait analysis with GWAS:

214 a review." Plant methods 9.1 (2013): 29

215 Lipka, Alexander E., et al. "GAPIT: genome association and prediction integrated 216 tool." Bioinformatics 28.18 (2012): 2397-2399. 
217 van Iterson, Maarten, Erik W. van Zwet, and Bastiaan T. Heijmans. "Controlling bias and inflation

218 in epigenome-and transcriptome-wide association studies using the empirical null

219 distribution." Genome biology 18.1 (2017): 19.

220 R Core Team (2016). R: A language and environment for statistical computing. R Foundation for

221 Statistical Computing, Vienna, Austria. URL https:/www.R-project.org/.

222 Sul, Jae Hoon, Lana S. Martin, and Eleazar Eskin. "Population structure in genetic studies:

223 Confounding factors and mixed models." PLoS genetics 14.12 (2018): e1007309.

224 Yang, Jian, et al. "Genomic inflation factors under polygenic inheritance." European Journal of

$225 \quad$ Human Genetics 19.7 (2011): 807.

$226 \mathrm{Yu}$, Jianming, et al. "A unified mixed-model method for association mapping that accounts for

227 multiple levels of relatedness." Nature genetics 38.2 (2006): 203.

228 Zhao, Keyan, et al. "Genome-wide association mapping reveals a rich genetic architecture of 229 complex traits in Oryza sativa." Nature communications 2 (2011): 467. 